\title{
Antitumor-Promoting Constituents from Dioscorea bulbifera L. in JB6 Mouse Epidermal Cells
}

\author{
Huiyuan GaO, ${ }^{a}$ Masanori Kuroyanagi, ${ }^{*, b}$ Lijun Wu ${ }^{a}$ Nobuo Kawahara, ${ }^{c}$ Takeaki Yasuno, ${ }^{d}$ and \\ Yoshiyuki NAKAMURA ${ }^{d}$ \\ ${ }^{a}$ Department of Traditional Chinese Medicines, Shengyang Pharmaceutical University; Shengyang 110016 China: \\ ${ }^{b}$ School of Bioresources, Hiroshima Prefectural University; 562 Nanatsukacho, Shobara, Hiroshima 727-0023, Japan: \\ ${ }^{c}$ National Institute of Health Sciences; 1-18-1 Kamiyoga, Setagaya-ku, Tokyo 158-8501, Japan: and ${ }^{d}$ School of \\ Pharmaceutical Sciences, University of Shizuoka; 52-1 Yada, Shizuoka 422-8526, Japan. \\ Received April 10, 2002; accepted May 27, 2002
}

\begin{abstract}
An antitumor-promoting effect was found in the extracts/ingredients of a plant used as a traditional medicine in mainland China, using the neoplastic transformation assay of mouse epidermal JB6 cell lines. The ethyl acetate soluble fraction of $75 \%$ ethanol extract of the rhizomes of Dioscorea bulbifera $\mathbf{L}$. showed an inhibitory effect against the tumor promotion of JB6 ( $\mathrm{Cl} 22$ and $\mathrm{Cl} 41)$ cells induced by a promoter, 12-O-tetradecanoylphorbol-13-acetate (TPA). Further investigation on the constituents of the EtOAc fraction from the rhizomes revealed the chemical structure to be kaempferol-3,5-dimethyl ether (1), caryatin (2), (+)-catechin (3), myricetin (4), quercetin-3-O-galactopyranoside (5), myricetin-3-O-galactopyranoside (6), myricetin-3-O-glucopyranoside (7) and diosbulbin $B(8)$. Constituent antitumor-promoting activities were also examined in the same way. Compounds $1-7$, characterized as flavonoids with the two hydroxyl groups at C-7 and C-4', showed the most potent inhibitory effect, but there seemed to be differences in the inhibitory effect between flavonol aglycones and flavonol glycosides. Compared with $(-)$-epicatechin, $(+)$-catechin exhibited much stronger inhibitory activity which suggested that chemical stereo structures of compounds affect the efficiency of inhibition. Compound 8 showed moderate activity. The constituents with antitumor-promoting activity from this plant are reported for the first time.
\end{abstract} side

Key words antitumor-promoting activity; JB6 cell; soft agar colony formation method; Dioscorea bulbifera L.; flavonoid glyco-

Much attention has been given to the primary prevention of cancer in daily life. ${ }^{1)}$ Suppression of the tumor promotion step by the functional constituents of plant resources is expected to prevent cancer development. The rhizome of Dioscorea bulbifera L. (Dioscoreaceae), a traditional Chinese medicine, has been used to treat sore throat and struma in ancient China. ${ }^{2}$ The plant is distributed in southeast Asia. People of the northern districts in Bangladesh use the rhizomes for treatment of leprosy and tumors, ${ }^{3)}$ while a Japanese patent was applied for to remedy diabetes in $1996 .{ }^{4)}$ To find novel antitumor-promoting agents from plant resources, the activity of extracts from one hundred plants was evaluated by the antitumor-promotion screening assay of JB6 mouse epidermal cell lines $(\mathrm{Cl} 22, \mathrm{Cl} 41)$ in vitro induced by 12-O-tetradecanoylphorbol-13-acetate (TPA, $1 \mathrm{ng} / \mathrm{ml}$ ). A $75 \%$ EtOH extract of the rhizomes of $D$. bulbifera $\mathrm{L}$. and the EtOAc soluble fraction of this extract showed very potent inhibitory effects on tumor promotion. Purification of the EtOAc fraction by chromatography and HPLC gave eight compounds and their structures were identified as kaempferol-3,5-dimethyl ether (1), caryatin (2), (+)-catechin (3), myricetin (4), quercetin-3-O-galactopyranoside (5), myricetin-3-O-galactopyranoside (6), myricetin-3-O-glucopyranoside (7) and diosbulbin B (8). This paper deals with the inhibitory effects of these constituents and discusses the relationship between the activity and the structures.

\section{MATERIALS AND METHODS}

General Experimental Methods NMR spectra were recorded on a Bruker-ARX $\left({ }^{1} \mathrm{H}-\mathrm{NMR} ; 300 \mathrm{MHz},{ }^{13} \mathrm{C}-\mathrm{NMR}\right.$;

$75 \mathrm{MHz})$ and a JEOL- $\alpha-500\left({ }^{1} \mathrm{H}-\mathrm{NMR} ; 500 \mathrm{MHz},{ }^{13} \mathrm{C}-\right.$ NMR; $125 \mathrm{MHz}$ ) spectrometer with tetramethylsilane (TMS) as an internal standard. Melting points were determined on a Yanaco Mp-S3 micro melting point apparatus and are uncorrected. HPLC separation was carried out on a reversed phase YMCR-ODS-5A packed column using the gradient $\mathrm{CH}_{3} \mathrm{CN}-$ $\mathrm{H}_{2} \mathrm{O}$ and $\mathrm{MeOH}-\mathrm{H}_{2} \mathrm{O}$ solvent systems, with detection at 210 $\mathrm{nm}$. Silica gel 60 (Merck) was used for column chromatography. JB6 cell culture was performed in a $\mathrm{CO}_{2}$ incubator $(\mathrm{Hi}-$ rasawa Works, Model WJ-22F)

Plant Materials Air-dried rhizomes of D. bulbifera L. were purchased from the hospital of Shengyang Rail, China in December 1999.

Extraction and Isolation Chipped rhizomes $(5 \mathrm{~kg})$ were extracted with $75 \% \mathrm{EtOH}$ to afford an extract (DB-75E, $1500 \mathrm{~g})$. An aliquot of $650 \mathrm{~g}$ of the fraction was suspended in water and partitioned successively with $\mathrm{CHCl}_{3}$ (DB-C), EtOAc (DB-A), $n$-BuOH (DB-B) to afford $8.2 \mathrm{~g}, 74.5 \mathrm{~g}, 55 \mathrm{~g}$ and aqueous residues (DB-W), respectively. DB-A (70 g) was

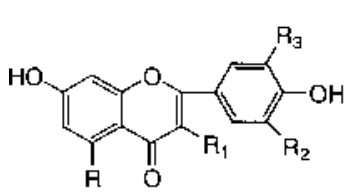

1 H- $-\mathrm{A}_{1}-\mathrm{OM}$. $\mathrm{F}_{2}-\mathrm{R}_{3}-\mathrm{H}$

$2 \quad \mathrm{R}=\mathrm{R} .=\mathrm{OHN}, \mathrm{R}_{2}=\mathrm{H}, \mathrm{A}_{3}=\mathrm{OH}$

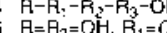

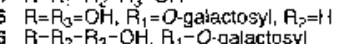

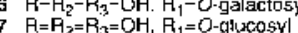

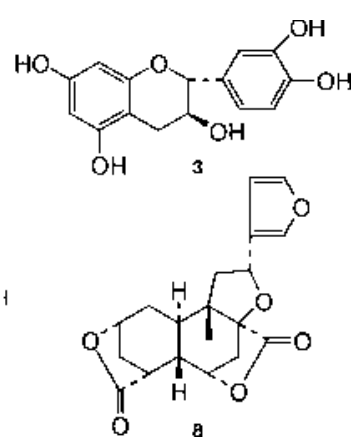


chromatographed on a silica gel column with a gradient solvent system $\mathrm{CHCl}_{3}-\mathrm{MeOH}$ to afford ten fractions $(\mathrm{F} 1-10)$. Fractions $3(0.5 \mathrm{~g}), 5(7.2 \mathrm{~g}), 6(1.5 \mathrm{~g})$ and $7(5 \mathrm{~g})$ were further separated by repeated silica gel columns using the $\mathrm{CHCl}_{3}-\mathrm{MeOH}$ solvent system, respectively, and the subfractions were further purified by HPLC. Compounds 1 (36 mg), $2(177 \mathrm{mg})$ and $3(2.4 \mathrm{~g})$ were isolated from F5. Compound 4 $(13.4 \mathrm{mg})$ was isolated from F6. Compounds $6(21 \mathrm{mg})$ and 7 (19 mg) were isolated from F7, and Compound 8 (214 mg) from F3. The structures of $\mathbf{1}-\mathbf{8}$ were identified by comparison of their reported ${ }^{1} \mathrm{H}$ - and ${ }^{13} \mathrm{C}-\mathrm{NMR}$ spectra data. ${ }^{5,6)}$

Chemicals TPA (Eden Prairie, MN, U.S.A.), MEM (Nissui Pharmaceutical Co., Ltd., Tokyo, Japan), fetal bovine serum (FBS) (Hazleton, PA, U.S.A.), Bacto agar (Difco Laboratories, MI, U.S.A.), and dimethyl sulfoxide (DMSO) (Dotite Spectrosol, Kumamoto, Japan).

Cells JB6 $\mathrm{Cl} 22$ and $\mathrm{Cl} 41$ cells were a kindl gift from Dr. N. H. Colburn (NCI, Frederick, MD, U.S.A.). The cells were cultured in $8 \%$ FBS-MEM medium at $36.5^{\circ} \mathrm{C}$ in an incubator with $5 \% \mathrm{CO}_{2}$ and $95 \%$ air.

Cytotoxicity Experiments JB6 cells ( $\mathrm{Cl} 22$ and $\mathrm{Cl} 41)$ seeded at a density of $1 \times 10^{4}$ cells per well in a 24 well plate were exposed to several doses of each sample with or without TPA $(1 \mathrm{ng} / \mathrm{ml})$ starting $20-24 \mathrm{~h}$ after seeding. After 3 or $4 \mathrm{~d}$ of treatment, cells while growing logarithmically were fixed with $10 \%$ formalin and stained with $0.1 \%$ crystal violet. The number of surviving cells was determined by microplate reader HTS-7000 of Perkin Elmer at $570 \mathrm{~nm}$. Maximum dose of sample used in the soft agar colony assay was set at over $90 \%$ at the cell survival rate.

Antitumor-Promoting Experiments in Vitro An antitumor-promoting activity was estimated by the inhibitory ability against the soft agar colony induction by TPA in JB6 $\mathrm{Cl}$ 22 and $\mathrm{Cl} 41$ cell lines as previously described. ${ }^{7)}$ Cells growing logarithmically in a monolayer culture were trypsinized and suspended in $0.33 \%$ agar medium containing $10 \%$ FBS and $1 \mathrm{ng} / \mathrm{ml}$ of TPA $\left(1.6 \times 10^{9} \mathrm{M}\right)$ with or without samples at the concentration indicated in Tables 1 and 2 . In duplicate 60 $\mathrm{mm}$ Petri dishes $1.5 \mathrm{ml}$ of the suspension containing $1 \times 10^{4}$ cells was plated over a bottom $0.5 \%$ agar layer containing the same concentration of TPA and/or samples. Soft agar colonies of 8 or more cells were scored after $14 \mathrm{~d}$. The inhibitory activities were expressed as \% of TPA-control with the average of two independent experiments using $\mathrm{Cl} 22$ and $\mathrm{Cl} 41$.

\section{RESULTS AND DISCUSSION}

In this antitumor-promoting screening system, TPA (1 $\mathrm{ng} / \mathrm{ml}, 1.6 \mathrm{nM})$ consistently induced anchorage-independent colony yields of $2695 \pm 563$ per $1 \times 10^{4}$ cells for JB6 Cl 22 $(n=14)$ and $2305 \pm 635$ per $1 \times 10^{4}$ cells for JB6 $\mathrm{Cl} 41$ $(n=14)$. Unless otherwise stated, the maximum concentration of transformation experiments did not inhibit cell growth in a monolayer. Tables 1 and 2 summarize the antitumor-promoting activities of the fractions and constituents from the rhizomes of D. bulbifera L.

Seventy-five percent of EtOH extract (DB-75E) from the rhizomes of $D$. bulbifera $\mathrm{L}$. showed the significant inhibition of TPA-induced anchorage independence in a dose-dependent manner. At the concentration of $30 \mu \mathrm{g} / \mathrm{ml}$, DB-75E decreased colony induction by $6.7 \%$ and $40.3 \%$ in $\mathrm{Cl} 22$ and $\mathrm{Cl}$ 41 , respectively.

Partition of DB-75E with $\mathrm{CHCl}_{3}$, EtOAc and $n$-BuOH successively gave DB-C, DB-A, DB-B and aqueous (DB-W) soluble fractions. DB-A and -B were found to contain very potent antitumor promoter(s), and almost completely inhibited the soft agar colony induction in JB6 cells at the concentration of $30 \mu \mathrm{g} / \mathrm{ml}$. The $50 \%$ inhibitory concentrations $\left(\mathrm{IC}_{50}\right)$ of them were $2.6 \mu \mathrm{g} / \mathrm{ml}$ and $4.4 \mu \mathrm{g} / \mathrm{ml}$, respectively. Further separation and identification of DB-A fraction by silica gel column chromatography and HPLC techniques gave eight compounds, $\mathbf{1}$ to $\mathbf{8}$.

Compounds $1-7$, which were identified as flavonoids with two hydroxyl groups at C-7 and C-4, showed remarkable antitumor-promoting activities. Especially, the flavonols of $\mathbf{1}, \mathbf{2}$ and $\mathbf{4}$ and catechin of $\mathbf{3}$, at the concentration of 10 $\mu \mathrm{g} / \mathrm{ml}$ and $30 \mu \mathrm{g} / \mathrm{ml}$, respectively, showed the most potent inhibitory effects on the TPA-induced soft agar colony formation by $0.64,02,8.2,6.2$, and $1.7 \%$ of TPA-control. The $\mathrm{IC}_{50}$

Table 1. Antitumor-Promoting Effect of the Fractions from D. bulbifera L. in JB6 Cell Lines

\begin{tabular}{lccc}
\hline \hline \multirow{2}{*}{ Fraction } & Max. dose tested, $\mu \mathrm{g} / \mathrm{ml}$ & \multicolumn{2}{c}{ Soft agar colony induction } \\
\cline { 3 - 4 } & & \% of TPA-control $^{a)}$ & $\mathrm{IC}_{50}, \mu \mathrm{g} / \mathrm{ml}$ \\
\hline DB-75E & 30.0 & 23.5 & 5.3 \\
DB-C & 10.0 & 61.4 & 18.0 \\
DB-A & 30.0 & 0.0 & 2.6 \\
DB-B & 30.0 & 7.4 & 4.4 \\
DB-W & 50.0 & 33.6 & 25.7 \\
\hline
\end{tabular}

a) All values are the averages of two independent experiments using JB6 cell lines of $\mathrm{Cl} 22$ and $\mathrm{Cl} 41$

Table 2. Antitumor-Promoting Effect of $\mathbf{1}-\mathbf{8}$ Isolated from Fraction DB-A in JB6 Cell Lines

\begin{tabular}{|c|c|c|c|}
\hline \multirow{2}{*}{ Compound } & \multirow{2}{*}{ Max. dose tested, $\mu \mathrm{g} / \mathrm{ml}$} & \multicolumn{2}{|c|}{ Soft agar colony induction } \\
\hline & & $\%$ of TPA-control ${ }^{a)}$ & $\mathrm{IC}_{50}, \mu \mathrm{g} / \mathrm{ml}(\mu \mathrm{M})$ \\
\hline Kaempferol-3,5-dimethylether (1) & 10.0 & 0.2 & $0.64(1.86)$ \\
\hline Caryatin (2) & 10.0 & 8.2 & $3.0(8.35)$ \\
\hline$(+)$-Catechin $(3)$ & 30.0 & 1.7 & $13.1(45.1)$ \\
\hline Myricetin (4) & 10.0 & 6.2 & $3.7(10.6)$ \\
\hline Quercetin-3-O-galactoside (5) & 30.0 & 38.7 & $21.6(43.6)$ \\
\hline Myricetin-3-O-galactoside $(\mathbf{6})^{b)}$ & 30.0 & 20.8 & $9.2(18.5)$ \\
\hline Myricetin-3-O-glucoside $(7)^{b)}$ & 30.0 & 20.8 & $9.2(18.5)$ \\
\hline Diosbulbin B (8) & 30.0 & 58.8 & $91.0(266)$ \\
\hline
\end{tabular}

a) Described in footnote of Table 1. b) The activity was tested using an equal mixture of 6 and 7 . 
of compounds $\mathbf{1}, \mathbf{2}, \mathbf{4}$, and $\mathbf{3}$ were 0.64 (1.86), 3.0 (8.35), 3.7 (10.6), and $13.1(45.1) \mu \mathrm{g} / \mathrm{ml}(\mu \mathrm{M})$, respectively. The data indicates that the activity of $\mathbf{1}$ was the strongest among compounds $\mathbf{1}$ to $\mathbf{4}$ as shown in Table 2 .

In contrast, compounds $\mathbf{5 , 6}$, and $\mathbf{7}$ exhibited relatively less potent activity. On the basis of structural characters, $\mathbf{1}, \mathbf{2}$, and $\mathbf{3}$ were flavonols, while compounds $\mathbf{5 , 6}$, and $\mathbf{7}$ were flavonol glycosides. The considerable reduction of inhibitory effect on the soft agar colony induction might have been due to the presence of sugar moieties. Compounds 6 and 7 were the glycosides of $\mathbf{4}$ binding the sugars at C-3 position, which was tested on antitumor-promoting activity using an even mixture of 6 and 7, and showed the inhibitory activity by $20.8 \%$ of TPA-control at the concentration of $30 \mu \mathrm{g} / \mathrm{ml}$, while compound 4 exhibited remarkably greater activity. The existence of sugar moieties might slow down the intracellular incorporation of the test compound. Difference of the molecular models of 1, 2 and 4 suggested that attachment of more hydroxyl groups at other positions (except C-7 and 4') could not contribute to the increase in ability of antitumor-promoting activity. Compound 3, identified as (+)-catechin, exhibited much more potent activity than (-)-epicatechin did (isolated from another plant). The inhibitory effects were $1.7 \%$ and $68.2 \%$ of TPA-control, respectively, at the concentration of $30 \mu \mathrm{g} / \mathrm{ml}$. On the other hand, (+)-catechin was less toxic than (-)-epicatechin in JB6 cells. Recently, Weyant et al. ${ }^{8)}$ reported that $(+)$-catechin inhibits colorectal cancer formation and suppresses focal adhesion kinase activation in mive. It is suggested that $(+)$-catechin has a different mechanism of action from the (-)-epicatechin derivatives. The difference in the biological activities of the stereoisomers suggested that stereo-structure might play an important role in the anticarcinogenic activity. Although diosbulbin B, one of the main constituents of the EtOAc layer, is known to show remarkable growth inhibition against the solid sarcoma 180 tumor in mice, ${ }^{9)}$ it showed only medium antitumor-promoting activity in JB6 cell lines without cytotoxicity at the concentration of $30 \mu \mathrm{g} / \mathrm{ml}$.

It is known that the mechanism in suppressiing JB6 cell tumor promotion induced by TPA is involved in modification of the signal transduction process, such as onco-protein kinase activation, reactive oxygen burst, and so on. ${ }^{10,11)}$ Therefore, a similar mechanism of action is assumed with the green tea catechin in the polyphenolic compounds identified from the rhizomes of D. bulbifera L. (Dioscoreaceae). Components with antitumor-promoting activities from $D$. bulbifera $\mathrm{L}$. were isolated in the experiment, and are reported for the first time. This result may be helpful in clarifying the reason that rhizomes of D. bulbifera $\mathrm{L}$. can be used in clinical treatment for human cancer.

\section{REFERENCES}

1) World Cancer Research Fund/American Institute for Cancer Research, "Food, Nutrition and Prevention of Cancer: a Global Perspective," American Institute for Cancer Research, Washington, 1997.

2) Jiang S., New medical college ed., "Dictionary of Chinese Crude Drugs," Shanghai Scientific Technological Publisher, Shanghai, 1978, p. 2059.

3) Robert D. H. M., Zacaris D. J., Nurul H. K., Mohammad S., Mohammad Q., Phytochemistry, 23, 623-625 (1984).

4) Akaha T., Jpn. Kokai Tokkyo Koho JP 08-325159 (1996).

5) Gao H., Lu Y., Wu L., Gao D., Journal of Shengyang Pharmaceutical University, 18, 185-188 (2001).

6) Gao H., Wu L., Yin K., Tang T., Journal of Shengyang Pharmaceutical University, 18, 414-416 (2001).

7) Nakamura Y., Colburn N. H., Gindhart T. D., Carcinogenesis, 6, 229235 (1985).

8) Weyant M. J., Carothers A. M., Dannenberg A. J., Bertagnolli M. M., Cancer Res., 61, 118-125 (2001).

9) Komori T., Toxicon, 35, 1531-1536 (1997).

10) Ahmad M., Mukhtar H., Nutr. Rev., 57, 78-83 (1999).

11) Nomura M., Ma W.-Y., Chen N., Bode A. M., Dong Z., Carcinogenesis, 21, 1885-1890 (2000). 